\title{
Algunos comentarios a los principios de París:
}

\author{
segunda parte
}

Ofelia Solis Valdespino

Investigadora del CUIB/UNAM

\section{RESUMEN}

Derivadelartículo an terior sobre los Principios de Ca talogación. Su pro pó si toesco men tar cada uno de los 12 prin ci pios de Catalogaciónresultantes de dicha Con fe ren cia, a la luz de los años trans cu rri do s y las experiencias concretas de la aplicación de ta les prin ci pios en la for mu la ción de có di gos ca ta lo gráfi cos en Eu ro pa y en los Es ta dos Uni dos. Fi nal men te se concuerda con la opinión de los teóricos en organización bibliográfica en cuanto a que son indudablemente valiosos para el quehaceres pe cífico deca taloga ción y de que es al ta men te de seable una nue vaconferen cia quelos me jore y ac tualice dados los fuertes cambios que ha introducido la automatización en las bibliotecas.

\section{ABSTRACT}

The study is derived from the previous article on the International Conference on Cataloging Principles. Its object is to comment on the 12 principles of cataloging that are the result of this conference, in the light of the years that have passed and the Uni ted Sta te Fi nally the se are inaccord with the of theo rists in bi blio gra phi calca ta loging or ga ni za tion in re gards to their un doub ted va lue in the spe ci fic task of ca ta lo ging and that a new con fe ren ce bet ter them and bring them up to date given the important changes that automation has introduced in libraries.

\section{INTRODUCCIÓN}

Este documento es una continuación al titulado "Recordando la Conferencia Internacional sobre Principios de Catalogación".

Tiene como propósito exponer una serie de observaciones originadas en la lectura de la Ex po si ción de Princi pios que se incluyo en el artículo anterior.
Para introducirse a la estructura general de la Exposición de Principios, debe tenerse claro que hay dos grandes áreas, a saber: principios que rigen en las reglas de catalogación para todo género de publicaciones, estos son los Prin ci pios 1 al 7; y las re glas que pue den apli car se a la ca taloga ción de pu bli ca cio nes par ti cu la res, que son los Principios 8 al 12 . 


\section{COMENTARIOS}

\section{Alcances de la exposición.}

Se establece que los Principios no pretenden constituir un código catalográfico completo. Esto es congruente con le objetivo de la Conferencia establecido en Londres en el mes de ju lio de 1959. "lle gar a un acuer do so bre los prin cipios fundamentales relativos a la elección y forma del encabezamiento en el catálogo alfabético de autores y títulos".

Los primeros están formados especialmentepara las grandesbibliotecas generales (seentien debiblio te cas nacionales, centrales de universidades, públicas con acervo importantes, etc.). Posiblemente hace 23 años las necesidades de estas bibliotecas. Asumiendo que así fuera, la licencia implícita para que otras bibliotecas sigan los Principios haciendo modificaciones en los encabezamientos de sus catálogos que puedan contradecir en algún momen to a los pro pios Prin ci pios, se gún sus muy par ti cu la res necesidades, conduce necesariamente a la falta de uniformi dad aun den tro de un mis mo ca tá lo go. Esto es con tra dictorio con el espíritu de la Conferencia. Si se busca lograr uni for midad, no de ben de jar se po si bi li da des de que se presenten variaciones significativas en los encabezamientos. Hay otro aspecto importante: en materia de cooperación interbibliotecaria las insignificantes variaciones locales resultan financiera, cooperativa y bibliográficamente inaceptables, sobre todo en nuestra era de interdependencia informativa a todos los niveles (local, regional e internacional).

Se aplican sólo a la elaboración de encabezamientos para catálogos de libros organizados alfabéticamente por autor y título. Por lo tanto, quedan fuera otros catálogos dentro de una misma biblioteca ${ }^{2}$

En cuanto a la terminología em plea da, el Vocabulariobásico de terminología de la Catalogación ${ }^{3}$ que se procuró observar durante la Conferencia, proporciona las siguientes definiciones:

Encabezamiento (heading): Palabra o grupo de palabras que se encuentran al principio de un asiento, que se distinguen del res to del asien to por su po si ción o ti po gra fía y determina el lugar del asiento en el catálogo.
Palabra ordenada (entry-workd): Palabra que determina el lu gar que un asien to o gru po de asien tos afi nes de ben tener en el catálogo.

Asiento (entry): Una de las anotaciones de las que se compo ne el ca tálogo, que pue de ser: asien to prin ci pal, asien tos secundarios o referencias.

Se circunscribe el tipo de material bibliográfico que está representado en el catálogo a uno solo: libro; admitiendo también aquellos materiales que presenten características semejantes a éste. Lo cual deja fuera casi todo el material no libro material que en buena medida contienen cada vez más las bibliotecas.

Se recomienda su aplicación a otros catálogos y "listas alfabéticas de libros con las modificacionesque exijalafinalidad de estos catálogos y listas". Aquí se refiere a catálogos delibreros y bibliografías. Se re co nocelaim portan cia de lo grar la ma yor uni for mi dad po sible des de el proceso de comercialización del libro para efectos de iden tificación unívoca; esteideal casi se halogra dogracias al uso cada vez mayor delNúmero In ternacional Normalizado para Libros (ISBN) a partir de los años setenta.

Sin em bar go, cabe se ña lar que la per mi sión de ha cer mo dificaciones dificulta la identificación de los libros. Por ejem plo: en unabiblio te ca na cio nal se asien tan los trabajos de Ovi dio bajo la for ma la ti na de su nom bre (Ovi dius); una bibliografía lo hace bajo la forma española del nombre (Ovidio); y una biblioteca pequeña bajo la forma que aparece en la portada de cada obra que de este au tor po sea, situación que no le reporta problemas debido a las dimensiones del acervo que contiene. ¿Qué pasa entonces con el ideal de nor ma li zar los nom bres para fa ci li tar el control de la producción libraria?

\section{Funciones del catálogo:}

Se ocu pa de los obje tivosfun da men tales del ca tálogoalfabético de autor-título, no de los medios para conseguir estos objetivos.

Se rea fir ma los pro pó si tos de Cut ter para el ca tá lo go y con esto se re for mu lan las di fe ren cias de opi nión en tre Pa nizzi y Cres ta do ro en el es tu dio del ca tálo go del Mu seo Bri tá nicoen 1849. El di le ma ha sido bus car la re con ci lia ción en tre el mecanismo requerido para la búsqueda de una obra espe cífi ca y la co lec ción de asien tos re la ti vos a una de obras, 
o sea, la búsqueda específica contra la búsqueda genérica. En la redacción de los Principios puede observarse que se ha dado preferencia a la función de la búsqueda genérica sobre la de materiales específicos. Los estudios de uso de catálogo indican que la mayoría de las búsqueda se hacen por materiales específicos. Así, lo más conveniente sería revisar los Prin ci pios y pres cri bir do bles asien to en los casos que pre sen tan conflic tos. Por úl ti mo, los sis te mas au tomatizados permiten el acceso a la información bajo cualquier for ma de nom bre o del tí tu lo de los do cu men tos, de esta manera puede resolverse la cuestión Panizzi-Cristadoro. ${ }^{4}$

En cuanto a la sección 2.1, puede observarse que limita al usuario en la rea li za ción de sus bús que da. Esto es muy claro cuan do se tra ta de lo ca li zar ma te rial so bre un tema es pecífico y no se sabe quiénes han escrito qué al respecto. Aun que en la sec ción 6.4 se re co mien da el uso de asien tos secun da rios bajo co labora do res y títu los, es ob vio que sino se recurre al catálogo temático, el usua rio no po drálo calizar un solo do cu men to que sa tis fa ga sus re que ri mien tos informativos.

La sección 2.2 fue calificada por algunos delegados en la Conferencia como menor importancia. ${ }^{5}$ Sin embargo, es in dis pen sa ble ya que los usua rios no siem pre es tán en condiciones de identificar una obra por la forma normalizada del nombre del autor, o por el título particular de la obra cuya edición se localiza en la biblioteca. Esto resulta más cier to en los ca sos de tra duc cio nes, obras li túr gi cas y do cumentos oficiales.

\section{Estructura del catálogo}

Este Principio está des ti na do a los proble mas que pre sen ta la identificación y determinación de la forma del nombre que será elegida como base para el encabezamiento de los au to res cu yas obras han sido tra du ci das a di ver sas len guas; a obras he chas en colaboración; an to lo gías, au to ría des conocida y a libros comúnmente por diferentes títulos como es el caso de al gu nas obras li te ra rias fa mo sas como: El cantar del Mío Cid, Las mil y una noches, La canción de Rolando, etcétera.
Las cau sas de que el nom bre de un au tor pre sen te va ria ciones son: ${ }^{6}$

- formas ortográficas diferentes:

XIMENEZ, XIMENEZ, JIMENEZ, GIMENEZ

- diferentes formas originadas en trasliteraciones distintas: TCHEKOV, TECEHOV, CHEKHOV, CHEHOV, CHEJOV

- diferentes formas linguisticas:

GIOVANNI BOCCACCIO, JOHAN BOCACCI, JEAN BOCCACE, JOHAN BOCHAS

- uso de formas completas e incompletas:

MOLIERE, POQUELIN DE MOLIERE

- cambio de estado civil

DeELIZABETHBARRETBARRETT aELIZABETH BARRETT BROWNING

- cambio de estado eclesiástico:

ENEA SIL VIO PICCO LO MINI (pasó a ser: PAPA PIO II)

- adquisición de un título de nobleza:

BENJAMIN DIRSAELI (pasó a ser: EARL OF EARL OF BEACONFIELD)

- renuncia a un título nobiliario:

de JONH GRIGG, BARON ALTRICHAM a JOHN GRIGG

- cambio de nombre.

De CASIUS CLAY a MUHAMMAD ALI

- uso de seudónimos:

TINTORETTO (cuyo nombre verdadero era:

JACOBO ROBUSTI)

EL PENSADOR MEXICANO (cuyo nombre verdadero era:

JOSE JOAQUIN FERNANDEZ DE LIZARDI)

En cuanto a la sección 3.22, es claro que la investigación del nombre del autor en las diversas fuentes biográficas, bibliográficas, periodística, etc., pue de darcomoresultado varias formas diferentes de un mismo nombre. Es aquí donde el catalogador deberá guiar su criterio dentro de las 
políticas de labiblio te ca a fin de es table cer laficha de au toridad de autor correctamente.

Respecto de la función de los colaboradores y los coautores se aceptó que: ${ }^{7}$

Colaborador (Collaborater): "Persona que colabora en una obra o es responsable de un aspecto de ella pero no de todo su contenido."

Coautor (joint author): "Persona o corporación que colabora en la obra de un autor".

Ninguna de estas definiciones aclaran en su totalidad cuál es la distinción entre la tarea de un coautor y cuál la de un colaborador. De esta falta de precisión se han derivadoserias confusiones que se reflejan en los códi gosfor mulados después de la Conferencia.

Actualmente, ya se ha establecido con claridad esta diferencia. Escamilla los define como sigue: ${ }^{8}$

"Coautor.- Es el au tor que es cri be una obra jun to con otro u otros, con la con tri bu ción de cada uno en con jun to y noseparadamente. (el subrayado es mío).

Colaborador.- es la persona que aporta, junto con otra u otras, su trabajo para la realización de una obra; por lo general, sus aportaciones son independientes y cada una de ellas va firmada con su nombre".

\section{Clase de asientos}

Aquí se define la cantidad de información que deben conte ner las tres cla ses de asien tos que con for man la es truc tu ra de los catálogos de autor - título: principales, secundarios y re fe ren cias. La de fi ni ción de cada uno de es tos tér mi nos, durante la Conferencia. Fue como sigue: ${ }^{9}$

Asiento principal (main entry): El asiento principal concerniente a cualesquiera libro o publicación periódica que se encuentren catalogados en la colección, que contiene toda la información necesaria para la identificación y ubicación de la obra.
Asien to secunda rio (addedentry) Cual quier asien to que no es el principal, que contiene información completa o parcial, de una unidad bibliográfica en particular.

Referencia(reference): asien to que en vía al usua rio del catálogo a otro encabezamiento.

La elec ción del asien to prin ci pal se basa en la pre mi sa de la sección 4.1, donde se establece que tal asiento debe ser un asiento completo, y por implicación, que los asientos secundarios pueden ser abreviados, excepto para los catálogos en forma de libros. Esta premisa vino a reforzar la prác ti ca de ha cer di fe ren tes fi chas para el jue go de un solo libro, misma que todavía pueden verse en los catálogosde muchas biblioteca.

Laen se ñan za de la ca ta lo ga ción en Méxi co du ran te las década de los cincuenta y sesenta también refleja esta situación.

Pue den ver se en los tex tos de en se ñan za de la épo ca las instrucciones para mecanografiar cada una de las diferentes fichas que constituían el juego para un solo título. ${ }^{10}$

Hacia finales de la década de los sesenta comenzó a popula ri zar se el uso de la "tar je ta úni ca" gra cias al em pleo de la reprografía en las bibliotecas.

Los principios 3 y 4 se formularon en la época de la precomputarizaciónen las bibliotecas y con dicionaronlos sistemas de automatización para la catalogación. Ha tenido que transcurrir más de una dé ca da a par tir de la in cor poración de la com pu ta dora a la prac ti ca ca ta lo gráfica, paraque haya un verdadero reconocimiento de que los sistemas de catalogación legible a maquina son mucho más que una versión automatizadadel catálogo manual. Un registro legible a maquina no es sólo una tarjeta catalográfica con clave de recuperación dentro de sí misma. Ofrece la recuperación rápi day efi cien te de lainfor mación bibliográfica solicitada usualmente; permite también recuperar información me dian te pun tos de ac ce so no con ven cio na les para un catálogo manual, esto es, bajo cualquier información contenida en los registros catalográficos legibles a máquinas: pero además proporciona la posibilidad de recuperación mediante la combinación de una variedad de puntos de acceso. 
Así, la idea de asiento principal expuesta en 4.1 es irrelevante para los actuales sistemas automatizados. Este concep to ya de be ría ha ber sido aban do na do des de el mo men to en que se trabaja en los departamentos de procesos técnicos con el sis te ma de fi cha única, amen de que los re gis tros catalográficos le gibles a máqui na pier den todosen tido.Sin embargo, continúan allí casi embalsamado en los códigos decatalogacióny en los propios sis temas au to matizados. ${ }^{11}$

\section{Empleo de Asientos múltiples.}

Este Principio está relacionado con la sección 3.2 (cuando un li bro re quie re de más de un asien to de bi do a cir cuns tancias diversas).

Lapri me ra fun ción del ca tálogo (véa se 2.1 $1^{\text {a }}$-2.1b) re quie re que se haga un asien to por el nom bre del au tor o por el tí tulo tal como fi gu ra en la por ta da del li bro que se está ca ta logando, esto se específica en 5.1.

La se gun da fun ción del ca tá lo go (véa se 2.2) se rea li za mediante el establecimiento de encabezamientos uniformes; és tos ase gu ran que to das las obras de un mis mo au tor, o todas las edi cio nes de una mis ma obra que den jun tas en el catálogo. Su definición es:

Encabezamiento uniforme (uniform heading): Forma de un encabezamiento que se adopta para usarlo en el catálogo, ya sea para un autor (personal o corporativo), para un título o para cualquier otro encabezamiento.

La sección 5.3 contribuye para que se realice la segunda función del catálogo.

Hay una in con sis ten cia en el uso del tér mi no "asien to". La redacción sugiere que debe entenderse por asiento lo que normalmente se entiende por tal, y además la función de referencia. Esta confusión semántica ha causado muchos equívocos en el desarrollo de códigos basados en estos Principios y en el estudio de los propios Principios. En el uso bi blio gráficosinglés nor mal, un asiento se re fie re a un documento o grupo de documentos; una referencia indica una for ma va rian te del nom bre de un au tor per so nal o corporativo, o del título de una obra y no tiene relación con ningúndocumento en particular. Así las diferentesversio- nes de la Biblia se asientan bajo "Biblia..." y no, como sugiere este Principio, bajo un encabezamiento derivado del títu lo tal como apa rez ca en el li bro sus ti tu yen do a una referencia formalizada. Esta diferencia crucial entre asiento y referencia fue reconocida por el Joint Steering Commitee para la re vi sión de RCA-1; es de gran im por tan cia prác ti ca porque así al usuario se le facilitará enormemente la recuperación de información dado que habrá congruencia interna en el catálogo en cuanto a qué tipo de información en con tra rá bajo un asien to, y cuál es la di rec ción que de berá se guir si se ac ce sa por me dio de una re fe ren cia o en vío. ${ }^{13}$

\section{Función de las diferentes clases de asiento}

Siguiendo las estipulaciones de 5.1 y 5.2, son necesarios dos asinetos para cada libro, a saber: autor y título. Ahora bien, para de ter mi nar cuál de las dos ha brá de con si de rar se como asiento principal, debe tenerse claro cuál de los dos modos de empleo del catálogo es más importante: encontrar li bros parti cu la res (pu bli ca dos), o en con trar obras particulares (que pueden encontrase incorporada en diversos documentos que forman publicaciones distintas y pueden tener particularidades de identificación diferentes): ${ }^{14}$ Estas búsquedas dependen de los intereses de los usuarios.

Si lo que se basa comúnmente son libros particulares, el encabe zamien to basa doen lain for mación como se presenta en el libro será más útil como asiento principal.

En caso contrario, el asiento se hará bajo encabezamiento uniforme, porque reunirá todos los asientos de libros que contengan la misma obra.

Durante la Conferencia se señaló que el asiento puede variar en un mismo catálogo según la naturaleza del libro en el caso de búsqueda de obras. Los factores que se consideraron en esa oca sión fue ron: si la obra in cor po ra da en el libro es o no de importancia his tóri ca o li te ra ria; y si el li bro ha sido es cri to ori gi nal men te en la len gua del país en el que se está ca ta lo gan do. Se acor dó, en ton ces, acep tar como regla general que el asiento deberá consistir en una forma única de su nombre; no hubo acuerdo en cuanto a aceptar como asiento principal para todas las ediciones de la misma obra un títulouniforme úni co sin em bar go, síse acep tó este título uniforme para las obras muy conocidas, por 
ejem plo: la Biblia, los Tri pi ta ka, los clá si cos anó ni mos, etcétera. En razón de lo anteriormente expuesto, la sección 6.1 admite dos alternativas: ${ }^{15}$

I. Asiento uniforme para un autor

II. Cuando el asien to se hace bajo el tí tu lo, pero éste cambia en cada edición:

(a) For ma ba sa da en la in for ma ción que el li bro pre senta, y con un asiento se cun da rio bajo un tí tu lo uni forme;

(b) Forma de asiento basada en un título uniforme, y con un asiento secundario bajo el título tal como se presenta en la obra que se está catalogando.

Los ca sos es pe cia les a que hace re fe ren cia 6.2 son aque llos en que una per so na es cribe in ten cio nal men te con dos nombres distintos diferentes trabajos, por ejemplo: novelas, poesías o cual quier otro gé ne ro li te ra rio y ade más obras de carácter científico. Obviamente, cuando se presenta casos de esta naturaleza, lo procedente es elaborar referencias cruzadas (relaciones).

La lec tu ra de este Prin ci pio cau sa con fu sión pues to que no sees tablece cla ra men te la di fe ren cia en tre las fun ciones de los asientos principales, los secundarios, las referencias (envíos) y las relaciones (referencias cruzadas).

\section{Elección del encabezamiento uniforme.}

En este texto se establece que la elección del nombre que sirve de base para el asiento principal ha de hacerse determinando cuál es la forma empleada más comúnmente por los autores, y no como lo estipulan códigos, la formale gal y más completa.

En esta sec ción tam bién se se ña lan las fuen tes a las que deberán atenderse en la elección de la forma empleada frecuentemente por las personas para publicar. Estas son: las portadas, referencias en las enciclopedias y diccionarios, la for ma en con tra da en obras his tó ri cas, bio gráfi cas y críticas y se da preferencia a la forma aparecida en las ediciones originales de los libros. Por supuesto, al traducir una obra tan to el tí tu lo como el nom bre del au tor pue den va riar. Por ello, se admite que el título uniforme o el nombre del au tor, en su caso, pue dan ba sar se en las tra duc cio nes cuando la forma original de los mismos no tienen probabilidades de ser bus ca da en el ca tá lo go. Así, se tie ne una de fen sa a las ob je cio nes que con fre cuen cia se le ha cen a los asien to uniforme, esto es, si una biblioteca contiene predominantemente obras extranjeras y éstas se hallan traducidas, no es posible que tal biblioteca haga los asiento principales con base en los títu los ori gi na les o en las for mas ori gi nales del nom bre de los au to res por que és tos no re sul tan fa mi liares a los usua rios de esta bi blio te ca; y si apa re cie ra una edición original en esta biblioteca, es lógica que el asiento para este libro se haga con base en las traduc cionesquede dicha obra se tenga en la biblioteca.

Es en este Principio donde se percibe con más claridad la falta de congruencia en el uso de la terminología.

Citemos las definiciones dadas anteriormente:

Encabezamientos (heading): Palabra o grupo de palabras que se encuentran al principio de un asiento, que se distinguen del resto del asien to por su po si ción por su ti po gra fía y determinan el lugar del asiento en el catálogo.

Encabezamiento uniforme, o tipo (uniform heading): Forma de un encabezamiento que se adopta para usarlo en el ca tálogo ya sea paraun au tor (per so nal o cor pora ti vo), para un título o para cualquier otro encabezamiento.

Palabra ordenada (entry word): Palabra que determina el lugar que un asiento o grupo de asiento afines deben tener en el catálogo.

Es notorio en estas definiciones la escasa precisión en el manejo de los conceptos. Por ejemplo: encabezamiento y palabraordenadoranomues traunadiferenciasignificativa en cuan to a qué son y cuál es su fun ción, se con fun den a los asientos propiamente dichos con las referencias aun cuando el tipo de información que contienen y la función que cumplen sean en la practica perfectamen te diferen ciables.

La importante distinción entre elección de asiento (selección del asiento principal y de asientos secundarios para una obra) y la forma del encabezamiento (selección de un encabezamiento uniforme por el cual una persona, organismo corporativo o una obra serán asen ta das en el ca tá logo) es de fec tuo sa. En RCA-2 se in ten tó, con bas tan teéxi to, hacer esta distinción y con esto se evitaron las trampas crea das por la con fu sión de elec ción y for ma de los Prin cipios. La catalogación descriptiva es vista ahora como un proceso en el que el catalogador primero crea una descripción básicaestándar, luego elige pun tos de ac ce so para esa descripción y finalmente selecciona formas uniformes 
para esos puntos de acceso; cada procedimiento se hace conforme a reglas catalográficas y catálogos de autoridad existentes. La disposición de los ca pítu los de RCA-2 es table ce clara men te este or den. No obs tante, debe ha cer senotar que este Principio representa considerables progresos en el es ta ble ci mien to del con cep to de asien to bajo la for ma más co mún del nom bre de una per so na, y por ex ten sión, de los nombres de organismos corporativos. ${ }^{16}$

\section{Autor personal único}

La sec ción 8.1 no da guías for ma les para la ca ta lo ga ción de libros de paternidad dudosa; siempre que una persona en particular sea generalmente considerada como autor, el asiento principal deberá ser hecho bajo su nombre.

La sección 8.2 admite el uso de una forma abreviada del nombre como encabezamiento uniforme contra el empleo de la forma completa de los mismos sólo si la forma abreviada es por la cual un autor es comúnmente identificado. Permite cierta flexibilidad en la elección entre dos nombres com ple ta men te dis tin tos em plea dos por la mis ma persona. La elección entre nombre verdadero contra seudónimo, nombre usado anteriormente contra nombre usadoposteriormente, en tre apellidoy títulonobiliario,entre nombre secular y título eclesiástico, se hará siguiendo el uso predominante de los nombres y no reglas fijas.

A la sección 8.2.1 puede agregarse que mientras no haya edicionesmodernas el encabezamiento basado en la información que consta en las portadas puede resultar poco apropiado, porque dichoencabezamien to podríadiferirdel nombre por el que un autor o una obra son corrientemente indentificados. ${ }^{17}$

Los enun cia dos de este Prin ci pio son una sim plifi ca ción de las com ple jas re glas para for mas del nom bre, las cua les están mejor ejemplificadas por las reglas de ALA de 1949. Gorman considera que los Principios 7,8 y 9 son los más singu la res y sig nifica ti vos lo gros de los Prin ci pios por que conducen a la catalogación hacia el ideal de Ranganathan: elaborar los encabezamientos que la mayoría de los usuarios del catálogo consultarán para un asiento en particular. $^{18}$

\section{Asiento bajo nombres de entidades}

La dis cu sión en tro no a si una en ti dad debe o no ser con siderada "autor", fue una de las más importantes de la Conferencia.

Hubo un de ba te en tre los paí ses se gui do res del có di go prusiano (quienes no aceptaban la autoría corporativa) y los partidarios del reconocimiento a las entidades como autores, posición de los anglosajones y los soviéticos. Los bibliotecarios alemanes aceptaron la utilidad de asientos corporativo por dos razones principales: la primera es que el tiempo había revelado deficiencias en los procedimientos para el asiento bajo títulos prescritos en las Instrucciones Pru sia nas; y la se gun da ra zón fue que la ma yo ría de las bibliotecas alemanas habían sufrido serios daños durante los bombardeos de la segunda Guerra Mundial y urgía su reconstrucción. En este contexto las cargas prioritarias de trabajo catalográficos no eran un factor que se viera muy afectado por las innovaciones en las Instrucciones. ${ }^{19}$

Las delegaciones alemana y austríaca, pese haber reconocido la utilidad de los asiento corporativos, se resistían a aceptarel concep to de au toría cor porativa. Conlafinalidad de satisfacer sus objeciones, la palabra "autor" fue excluida de esta sec ción. Sin em bar go, la ver sión pre li mi nar de la exposición de Principios comprendía el concepto general de la co lec ti vi dad como au tor, y en el cur so de las dis cu siones, así como, en el texto final de la Exposición de Principios adoptada por la Conferencia no existe una sola re fe ren cia que in di que la ex clu sión de este con cep to ge neral.En ra zón de es tos con si de ran dos, en las sec cio nes 1 a 8 de la Exposición debe in ter pre tar se que la de fi ni ción de autor incluye a las en ti da des cu yos nom bres pue den serutilizados como asientos. ${ }^{20}$

La sección 9.1 muestra claramente el conflicto entre quienes acep tan que una en ti dad pue de ser au tor, y aque llos que no acep tan este ca rác ter pero que, sin em bar go, re co no cen que el nom bre que se pro por cio na en el tí tu lo de una pu blicación "es un medio cómodo para identificar la obra en el catálogo". ${ }^{21}$ La primera tendencia se manifestó en la prime ra edi ción de lasRe glas de Ca ta lo ga ción An glo-americanas que definen autor como: "El individuo o la entidad principalmente responsable de la creación del contenido intelectual o artístico de una obra". ${ }^{22}$ 
La segunda tendencia modificó el código alemán; así, la nue va re gla bási ca para colec ti vi da des publica das en 1965 por la ve rein Deuts cher Bi blio theka re in di ca que es ini ciadora de una obra puede servir de asiento principal solamente si la obra es anónima, o si el nombre de esta en ti dad está com pren di do en el tí tu lo de la obra, o si la obra no puede ser designada apropiadamente sin este nombre. ${ }^{23}$

La subsección 9.5 requiere de algunas observaciones: primero: si las constituciones, leyes y tratados son la expresión del pen sa mien to de una en ti dad te rri to rial, la elec ción del encabezamiento para el asien to principal ha debasarse en las sub sec cio nes 9.1.1 y 9.4.4; por lo tan to, esta sub sección proporciona la regla complementaria que indica que elencabezamiento debe ir seguido de un título convencional y no del títu lo que pre sen ta el do cu men to. Se gun do. En muchos catálogos se usa la ex pre sión "Le yes, de cre tos, etcétera" para reunir diversos materiales legislativos; pero esta expresión no puede ser considerada como un título "formal o convencional", ni como reemplazado del título de cada documento. Su uso describe colectivamente un gru po de obras que de ben es tar re u ni das en el ca tálo go, por lotan to es más apro pia do lla mar les su ben ca be za mien tos y no "“títulos formales o convencionales".

Res pec to a la sub sec ción 9.6 pue e de cir se que ha sido efectivamente, un principio seguido en la primera edición del Código Angloamericano, tal como se expresa en la Introducción a la Regla 78.

\section{Autores múltiples.}

La cláusula relativa a colecciones incluye la única instancia de un textoalternativo en los Principios. Estaal ternativa estipula un asiento para una colección bajo el compilador "si está nombrado en la portada ". RCA-1 siguió este textoalternativo. Hay que hacer notar que la discusión de este Principio gira en torno de un punto irrelevante: cuando un trabajo debería asentarse bajo el compilador con un asiento se cun da rio bajo tí tu lo y cuán do debería seguirse la práctica contraria.

Ade más se aban do na el tra ta mien to de cues tio nes más pertinente de cuando y con cuánta frecuencia deben hacerse asientos analíticos para las partes de cada trabajo u obra compues ta. Este úl ti mo pun to ha veni doin cre men tan do su im por tan cia; to da vía no ha sido tra ta do a fon do en nin gu na regla del Código Angloamericano. La razón obvia para estaca ren cia es la gran di fe ren cia de circuns tan cias y necesidades de los diferentes sistemas bibliotecarios. Sin embargo, muchos catalogadores enfrentan, día a día, el proble ma de no sabercuán tos asien tos ana líti cos de benhacer para una obra de autoría compartida, no cuál sería la forma precisa que dichos asientos deberían presentar. ${ }^{24}$

\section{Obras catalogadas por el título.}

Hay numerosos problemas asociados con este Principio, regresaremos luego sobre éstos, pero existen tres puntos que ameritan ser considerados aquí.

El prime ro, es la re co men da ción del asien to adi cio nal bajo título que tien de a se guir la lí nea eu ro pea y por eso pres cribe cuantos menos asiento de título que se estimen convenientes en la mayoría de las bibliotecas. Lo observado en es tu dios so bre el uso de ca tálo gos in di ca que el ac ce so bajo título es un atributo altamente deseable en los catálogos modernos.

Se gun do, la men ción in ci den tal de las se ries en el Prin ci pio 11.4 establece que el asiento por título se prescribe para "obras (in clui das las se ries y pu bli ca ciones pe rió di cas)conocidas principal o convencionalmente por título bastante más que por el nom bre del au tor." De jan do de lado la vas ta cues tión de sicuáles prin ci pioscategorizaríanlas ex tre madamente vagas "obras" más exactamente, un problema central en to dos los códi gos ca ta lo gráficos (asien to principal para series) permanece parcialmente tratada, y cada término general propone de casi cualquier opinión acerca del asiento de las series puede encontrar soporte en estos principios.

Tercero, el lenguaje de 11.6 suscita la cuestión que nos ocupará ahora. Aprueba los asientos "bajo un encabezamientoconvencional uni for me ele gi do para re fle jar la formade la obra" para trata dosinternacionales, convenciones y "ciertas categorías de publicaciones."Este propósito establece vagamente los márgenes a la secuela de formas de encabezamientos y ha guiado a muchos interpretaciones erróneas en los códigos catalográficos desde su formulación. 
M. Gorman opi na que no pue de exa ge rar se la im por tan cia de acla rar que las for mas de los en ca be za mien tos no tie nen lugar en un catálogo de autor - título de mencionar que tales prescripciones no deberían estar incluídas en estos Principios, y que el Principio 11 debería ser suprimido en una versión revisada de los Principios. ${ }^{25}$

\section{Palabra inicial para el encabezamiento de los nom- bres personales.}

Se ocu pa de la cues tión de los ele men tos de asien to para un en cabe za mien to para un au tor per so nal que con sis ta de dos o más pa la bras, y es ta ble ce que en cada caso el asien to debería basarse en la nacionalidad de la persona. Esto es un cri te rio irre le van te y con fre cuen cia es bas tan te difícil averiguar la nacionalidad de los autores.

El enunciado del Principio 12 podía ser reemplazado por un prin ci pio ge ne ral para el asien to de per so na ba sa do en el uso de la lengua que usa principalmente la persona. ${ }^{28}$

\section{CONCLUSIONES}

A poco más de 20 años de la formulación de estos principios, se hace necesario señalar algunas cuestiones:

- Sería conveniente abolir el párrafo donde se menciona que los Prin ci pios se des ti nan a ca tá lo gos de gran des bi- bliotecas y de jar que ten ga apli ca ción en todo tipo de bibliotecas, catálogos de libreros y otras listas bibliográficas.

- La re vi sión de la era com pu ta ri za da de los Prin ci pios relegar discusiones de la estructura de una lista impresa y de tipo de asien to den tro de ésta a una po si ción se cun daria y debe considerar las implicaciones de accesos multidimensionales a los datos. Además estas implicaciones de ben to mar en con si de ra ción la pre paración del catalogador para ingresar datos en un sistema com pu ta ri za do y la co di fi ca ción y el ran go de los pun tos de acceso en un registro complejo.

- En la empresaca ta lográfica aún hay ideas an ticuadas, es ur gen te la ne ce si dad de ree va luar las ba ses de ca ta lo ga ción a la luz de los desarrollo técnicos y teóricos.

- Los Principios contienen muchos errores de omisión y comisión. Su estilo es vago generalmente. Por lo tanto, no puede erigírseles como bases para adecuados códigos catalográficos en esta época de computación y bibliotecas con multimedia.

- Hay numerosos documentos en los que se señalan incon gruen cia y cues tio nes a op ti mi zar den tro de los Principios. La IFLA debería organizar una nueva Conferencia en donde todas las cuestiones que han sido detectadas se discutan y se llegue a nuevos acuerdos acor des a la épo ca en que vi vi mos y para los años por venir.

\section{CITAS BIBLIOGRÁFICAS}

CONFERENCIA INTERNACIONAL SOBRE LOS PRINCIPIOS DE CATALOGACIÓN (1961: PARIS) "Informepreliminar oficial". -En Boletínde la Unescoparabibliotecas,_-Vol. XVI, no.2 (mar zo-abril 1962) p.57.

_-Exposición de principios/ Edit. Por A.H. Chaplin con la asistencia de Dorothy Anderson. -Ed. prov. Tr. de la vers. francesapor Aída Opazo. - Caracas: Univ. Central de Venezuela, 1969 . h.1.

_- Vocabulariobásicodetérminologíadelacatalogación:anteproyectorevisadoe nlaConfe rencia/Tr. de Ma. Teresa Chávez Campomanes. - - [s.1.]... IFLA, [1962?] P.11.

GOR MAN, Mi chael. "Chan ges in ca ta lo guing co des: ru les for entry and hea ding”. -En library trends. - - No. 25 (jan. 1977) p.591.

CONFERENCIA INTERNACIONAL...Exposición ...h.[2] 
(6)
Ibid. h. 3

CONFERENCIA INTERNACIONAL ... Vocabulario ...p.9.

ESCAMILLA GONZALEZ, Gloria. Interpretación catalográficade los libros. - México : UNAM, Instituto de Investigaciones Bibliográficas: Facul tad de Filo sofía y Letras, 1979. - - (Ins trumentabibliographica;4: Manuales; 1) p.105.

CONFERENCIA INTERNACIONAL ...Vocabulario...p.5-6.

CHAVEZ CAMPOMANES, Ma. Teresa. Manual para catalogadores y clasificadores. - - México: Escuela Nacional de Bibliotecarios y Archivistas, 1960. P. 14-29.

GORMAN, p.592.

CONFERENCIA INTERNACIONAL ... Vocabulario...p.6.

GORMAN. P.593.

CONFERENCIA INTERNACIONAL...Exposición...h.7.

Ibid, h.7-14.

GORMAN. P. 593-594.

CONFERENCIA INTERNACIONAL ...Exposición...h.22.

GORMAN. P.594.

CARPENTER, Michael. Corporate authorship: its role in library cataloguing.-Westport, Conn: Greenwood, c1981._-(Contributions in librarianship and information science; no. 34) p.64.

CONFERENCIA INTERNACIONAL...Exposición ...h.24.

Loc cit.

Reglasdecatalogaciónangloamericanas:textonorteamericano/pre para das porThe Ame rican Library Associa tion...et al ; tr. au to ri za da, vers. al es pa ñol de Hor ten sia Agua yo, ase so ría y adaptación de Jorge Aguayo. - Washington: OEA, 1970. - (Manuales del bibliotecario; no. 7) p.334.

CONFERENCIA INTERNACIONAL...Exposición...h. 24-25.

GORMAN, p.594.

Ibid. p. 595 . 